Unfallchirurg 2013 · 116:6-7

DOI 10.1007/s00113-012-2298-5

Online publiziert: 21. Januar 2013

(c) Springer-Verlag Berlin Heidelberg 2013

\section{Münzberg' ${ }^{1}$ M. Perl ${ }^{2}$ D. Depeweg ${ }^{3}$}

${ }^{1}$ Klinik für Unfallchirurgie und Orthopädie, BG Unfallklinik Ludwigshafen

${ }^{2}$ Klinik für Unfallchirurgie, Hand- und Plastische Chirurgie, Universitätsklinikum Ulm

${ }^{3}$ Klinik für Orthopädie, Unfallchirurgie und Paraplegiologie, Universitätsklinikum Heidelberg

\title{
Junges Forum der Deutschen Gesellschaft für Orthopädie und Unfallchirurgie
}

Das Junge Forum der DGOU sieht sich als „Interessensvertretung“ junger Kolleginnen und Kollegen - vom Studium bis zum Facharzt und darüber hinaus. Ziel des Jungen Forums ist es, das Fach Orthopädie \& Unfallchirurgie (O \& U) für junge Kollegen attraktiver zu gestalten und für die wichtigen Belange im Fach $\mathrm{O} \& \mathrm{U}$ einzutreten.

Fast 10 Jahre ist es her als unsere Alumnikollegen die Idee hatten ein Junges Forum zu gründen, um so Ihre Zukunft selbst mit zu gestalten. Damals gab es noch zwei getrennte Fächer - Unfallchirurgie und Orthopädie. Die Probleme und die Herausforderungen in beiden Fächern sind heute nicht weniger als damals - um so glücklicher sind wir, dass uns unsere Alumnikollegen ein hervorragendes etabliertes und gut strukturiertes Netzwerk hinterlassen haben.

Auf unseren jährlichen Klausurtagungen kristallisierte sich seitdem wiederholt heraus, wo genau die Herausforderungen der heutigen Zeit liegen: Die Weiterentwicklung einer attraktiven und sinnvollen Weiterbildung im gemeinsamen Fach $\mathrm{O} \& \mathrm{U}$, die Realisierbarkeit von Familie und Beruf, die Fortführung einer kontinuierlichen Nachwuchsgewinnung, die Vereinbarkeit von Wissenschaft und zunehmend verdichteter klinischer Tätigkeit und nicht zuletzt die Aufgabe die immense Fülle der Inhalte des Faches O \& U durch Generalisten und/oder Spezialisten sinnvoll klinisch abzubilden.

\section{) Nachwuchsförderung \\ braucht Perspektiven \\ in einer strukturierten \\ modernen Weiterbildung}

Unsere Umfrage im letzten Jahr unter über 700 Kollegen/-innen in Weiterbildung unterstrich diesbezüglich die Dringlichkeit dieser Themen und mahnte zu weiterer kontinuierlicher Arbeit an den Problemlösungen. So wurde schnell klar, dass wir diese immensen Aufgaben nur mit einem größeren Team und einer neuen Struktur bewerkstelligen werden können. Daher wurden 2012 fünf Sektionen unter dem Dach des Jungen Forums etabliert: Weiterbildung, Familie und Beruf, Nachwuchsförderung, Wissenschaft, Öffentlichkeitsarbeit und Internet. Diese Struktur und die damit verbundenen Schwerpunkte des Jungen Forums der Deutschen Gesellschaft für Orthopädie und Unfallchirurgie (DGOU) sollen in diesem Heft zur Geltung kommen. Diese heute wichtigen Themen schließen unserer Meinung auch einen wichtigen Kreis zur Sicherung von ausreichend qualifiziertem Nachwuchs in unserem Fach in der Zukunft. Die derzeit sich in aller Munde befindliche Thematik Nachwuchsförderung braucht Perspektiven. Perspektiven in einer struk- turierten modernen Weiterbildung. Perspektiven in einer ausgewogenen Arbeitsatmosphäre mit der Realisierbarkeit von Familie und Beruf. Perspektiven mit der Möglichkeit für junge motivierte Kollegen das interessante Feld der Wissenschaft und der klinischen Ausbildung in einer angemessenen Zeit zu vereinbaren. Und nicht minder alltagstaugliche Perspektiven nach der Facharztausbildung.

Ein weiterer wesentlicher Aspekt, der hier zum Ausdruck gebracht werden soll ist die Gemeinsamkeit von $\mathrm{O} \& \mathrm{U}$. Wir sehen uns als das Junge Forum der DGOU - als die Vertretung angehender und junger Kollegen im Fach Orthopädie UND Unfallchirurgie. Ein Fach mit einer großen Geschichte, mit einer faszinierenden und unglaublichen Vielfalt und hoffentlich einer genauso großen Zukunft. Jegliche Bestrebungen einer Aufweichung der mühsam geschaffenen gemeinsamen Strukturen können nicht im Interesse der neuen Generation sein. Die Gemeinsamkeit muss auf allen Ebenen gelebt werden - von Studenten, von Assistenzärzten, von Oberärzten und von Chefärzten. Es gibt nur noch „O und U“.

Dass unsere Arbeit ernst genommen wird, sehen wir in der Tatsache, dass wir seit dem 01.01.2013 offizieller Ausschuss der DGOU sind und damit auch dem Geschäftsführenden Vorstand der DGOU zuarbeiten dürfen. Für die Möglichkeiten die uns bisher gegeben wurden 
an der Gestaltung unseres Faches mitzuwirken und für die Perspektiven, die uns nun hiermit eröffnet werden, möchten wir uns bei allen, die uns bis dato unterstützt haben, herzlich bedanken. Wir freuen uns, die Arbeit und die Visionen der Gründungsmitglieder des Jungen Forums für ein gemeinsames und schlagkräftiges O \& U fortsetzen zu können.

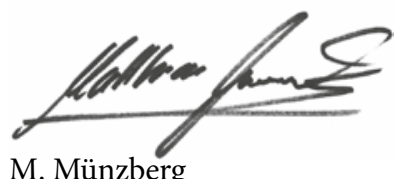

M. Münzberg

(Leiter Ausschuss Junges Forum DGOU)

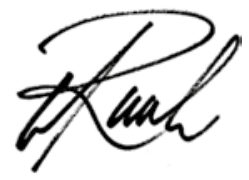

M. Perl

(Stlv. Leiter Ausschuss Junges Forum DGOU)

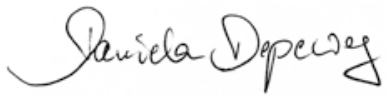

D. Depeweg

(Alumni Junges Forum DGOU)

\section{Korrespondenzadressen}



Dr. M. Münzberg

Klinik für Unfallchirurgie und Orthopädie,

Berufsgenossenschaftliche Unfallklinik Ludwigshafen Ludwig-Guttmann-Straße, 67071 Ludwigshafen matthias.muenzberg@bguludwigshafen.de

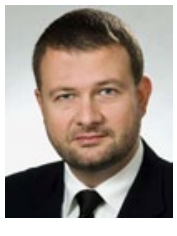

Prof. Dr. M. Perl

Klinik für Unfall-, Hand-, Plastische und Wiederherstellungschirurgie, Universitätsklinikum Ulm Albert-Einstein-Allee 23, 89081 Ulm marioperl@web.de

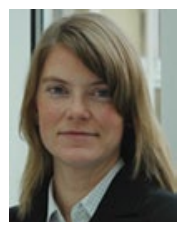

Dr. D. Depeweg

Klinik für Orthopädie, Unfallchirurgie und Paraplegiologie, Universitätsklinikum Heidelberg Schlierbacher Landstraße 200a, 69118 Heidelberg daniela.depeweg@med.uniheidelberg.de

\section{Ärzte Zeitung digital: Heute lesen, was morgen in der Zeitung steht}

\section{Im Publikumsbereich erfreuen sich App-Ausgaben von Zeitungen und Zeit- schriften wachsender Beliebtheit. Ab sofort ist auch Deutschlands einzige Ta- geszeitung für Ärzte in diesem Format erhältlich: die „Ärzte Zeitung digital“"}

„Ärzte Zeitung digital" bietet gesundheitspolitische Nachrichten, Kommentare, Hintergründe zu Medizin und wirtschaftlichen Themen rund um die Praxis - im übersichtlichen und intuitiven Lesemodus, optimiert für den Tablet-Computer. Die Vorteile für Leser der AppAusgabe der "Ärzte Zeitung":

- Die Ausgabe ist schon am Vorabend verfügbar, Leser können damit die Nachrichten des kommenden Tages bequem auf dem Sofa empfangen.

- Wichtige Nachrichten werden als „Breaking News" direkt auf das Tablet geleitet und lassen sich über die sogenannte Newslasche anzeigen.

- Die App ist so konzipiert, dass die gesamte Ausgabe im Hintergrund heruntergeladen werden kann. Anders als im Internet ist es für Leser dann möglich, sich offline durch die Ausgabe zu „blättern“, ohne Wartezeiten beim Durchklicken auf einer Website.

- Die alten Ausgaben bleiben zwei Wochen auf dem Tablet-PC verfügbar.

„Ärzte Zeitung digital" ist Teil des e.Med-Pakets von Springer Medizin. Zugänglich ist die App über den iTunes-Store von Apple - allerdings nur für registrierte Nutzer von Springer Medizin, die e.Med für 30 Tage kostenlos testen wollen, oder für e.Med-Abonnenten - und zwar ohne weitere Zusatzkosten. Die Version für Android-Tablets wird Anfang 2013 verfügbar sein.

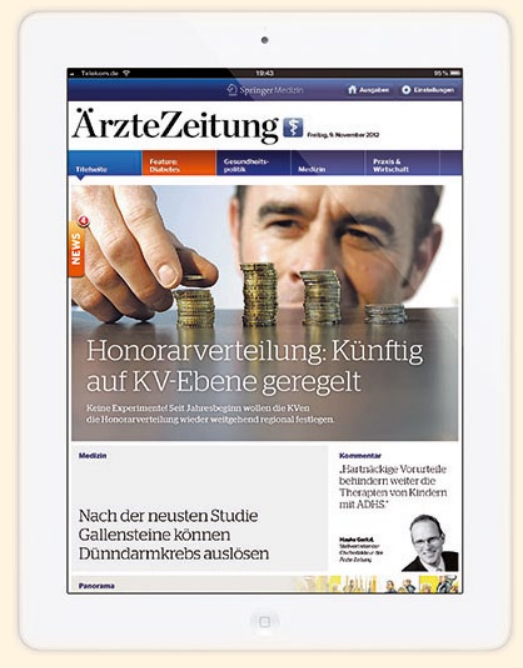

Abonnenten des e.Med-Pakets haben Zugriff auf rund 500 Fortbildungskurse in der e.Akademie, auf die Volltexte von mehr als 400 deutschsprachigen und internationalen Zeitschriften in der e.Bibliothek, auf einen Webbaukasten für die Praxiswebsite und ein PrintAbo einer Springer-Fachzeitschrift nach Wahl (plus Versandkostenpauschale). Das e.Med-Paket kostet 33,25 Euro im Monat.

Anmeldung zum kostenlosen e.Med-Testzugang: www.springermedizin.de/eMed/

Mehr Info zur App „Ärzte Zeitung digital“: www.springermedizin.de/tablet

Quelle: Ärzte Zeitung 